\title{
Aberrant TIMELESS expression is associated with poor clinical survival and lymph node metastasis in early-stage cervical carcinoma
}

\author{
WEIJING ZHANG $^{1 *}$, WEILING HE ${ }^{2 *}$, YONGJIE SHI $^{3 *}$, JING ZHAO $^{1}$, SAILAN LIU ${ }^{1}$, \\ FENGXIANG ZHANG ${ }^{4}$, JIARUI YANG ${ }^{4}$, CHUANMIAO XIE $^{1}$ and YANNA ZHANG ${ }^{1}$
}

\begin{abstract}
${ }^{1}$ Sun Yat-Sen University Cancer Center, State Key Laboratory of Oncology in South China, Collaborative Innovation Center for Cancer Medicine, Guangzhou, Guangdong 510060; ${ }^{2}$ Department of Gastrointestinopancreatic Surgery, The First Affiliated Hospital of Sun Yat-Sen University, Guangzhou, Guangdong 510080; ${ }^{3}$ Department of Clinical Examination, The Second Affiliated Hospital of Guangzhou Medical University, Guangzhou, Guangdong 510260; ${ }^{4}$ Eight-year program, Zhongshan Medical School, Sun Yat-Sen University, Guangzhou, Guangdong 510080, P.R. China
\end{abstract}

Received September 5, 2016; Accepted November 22, 2016

DOI: $10.3892 /$ ijo.2016.3784

\begin{abstract}
TIMELESS is a highly conserved protein required for the maintenance of normal mammalian circadian oscillations and for controlling cellular metabolism and proliferation. Recently, TIMELESS was implicated in the tumorigenesis of certain cancers. However, little is known on TIMELESS protein expression and its potential as a prognostic factor in cervical cancer. Here, we investigate TIMELESS expression pattern and its clinicopathological significance in early-stage cervical carcinoma. TIMELESS mRNA and protein expression was evaluated by real-time PCR and western blot analysis in cervical cancer cell lines, a normal cervical cell line, as well as in six pairs of surgically removed cervical cancer and adjacent normal cervical tissues. A total of 189 paraffinembedded cervical carcinoma specimens were detected and diagnosed by immunohistochemistry (IHC), and the clinical significance of TIMELESS expression was further analyzed. Aberrant TIMELESS mRNA and protein expression were demonstrated in cervical cancer cell lines compared with the normal cervical cell line. TIMELESS mRNA and protein expression were significantly increased in cervical cancer specimens compared with adjacent non-cancerous cervical
\end{abstract}

Correspondence to: Dr Yanna Zhang or Dr Chuanmiao Xie, Sun Yat-sen University Cancer Center, State Key Laboratory of Oncology in South China, Collaborative Innovation Center for Cancer Medicine, No. 651, Dongfeng Road East, Guangzhou Guangdong 510060, P.R. China

E-mail: zhangyn@sysucc.org.cn

E-mail: xiechm@sysucc.org.cn

*Contributed equally

Key words: TIMELESS, pelvic lymph node metastasis, lymphovascular space involvement, cervical cancer, prognosis, biomarker specimens. TIMELESS protein expression was significantly associated with the age $(\mathrm{P}=0.011)$, clinical stage $(\mathrm{P}<0.001)$, pelvic lymph node metastasis $(\mathrm{P}<0.001)$, squamous cell carcinoma antigen $(\mathrm{P}=0.003)$, tumor recurrence $(\mathrm{P}=0.015)$, vital status $(\mathrm{P}<0.001)$, tumor differentiation grade $(\mathrm{P}<0.001)$, property of the surgical margin $(\mathrm{P}=0.036)$ and lymphovascular space involvement $(\mathrm{P}=0.001)$. Patients with increased TIMELESS protein expression showed strong tendencies to receive postoperative radiotherapy $(\mathrm{P}=0.002)$. Upregulation of TIMELESS correlated with poorer overall survival (OS) and disease-free survival (DFS). Univariate and multivariate Cox-regression analyses showed that TIMELESS can be regarded as an independent predictive biomarker for poor clinical outcome for early-stage cervical carcinoma. Our results show that TIMELESS overexpression correlates with pelvic lymph node metastasis, lymphovascular space involvement, as well as unfavorable OS and DFS in human cervical cancer. Therefore, TIMELESS expression may be a potential prognostic biomarker for cervical cancer patients.

\section{Introduction}

Cancer of the uterine cervix is one of the most prevalent malignant neoplasms diagnosed, and remains the fourth main cause of cancer-related mortality among women worldwide (1). Globally, 529,800 new cases of cervical carcinoma are diagnosed each year, resulting in $\sim 275,100$ deaths annually, especially in developing countries (2). Infection with high-risk human papillomavirus (HPV) types is the common cause of cervical cancer, which leads to activation of oncogenes and deactivation of tumor suppressor genes (3). Smoking, multiple sexual partners, and poor sanitation are additional risk factors closely associated with cervical cancer development (4). Although advances in vaccinations, Pap smear screening programs, and therapeutic methods (including surgery techniques, chemotherapy and radiotherapy) have decreased the morbidity and mortality of cervical carcinoma $(5,6)$, these prevention, diagnostic tools and treatments have their 
limitations. Moreover, patients with lymph node metastasis, lymphovascular space involvement, and higher differentiation grade or FIGO stage show poorer clinical outcomes (7-9). While these prognostic indicators have been correlated with biomarkers (such as C14ORF166, B3GNT3, URG4 and CISD2) (10-14), we still require novel prevention and treatment strategies to improve the outcomes of patients with cervical cancer.

One potential therapeutic or prognostic target is TIMELESS, a 138,658-Da protein that was first characterized in Drosophila melanogaster as a core component of the circadian clock (15). Since then, TIMELESS has been shown to be a highly conserved protein that is also involved in the cell cycle checkpoint system, cell survival after damage or DNA replication stress, increasing DNA polymerase epsilon activity, maintaining telomere length, epithelial cell morphogenesis and in the formation of branching tubules $(16,17)$. Recently, circadian rhythm disruption was hypothesized to explain an increased susceptibility to certain malignancies (18). Indeed, previous studies report a relationship between TIMELESS and human cancers of many different organs, including lung, breast, liver, prostate, colon, kidney, bladder, pancreas and blood (19-31). However, the characteristics of TIMELESS expression in human cervical cancer have not been investigated and its clinical significance remains largely unknown.

In the present study, we sought to investigate the expression of TIMELESS in cervical cancer and analyze its clinicopathologic and prognostic value in 189 archival early-stage cervical carcinoma specimens.

\section{Materials and methods}

Cell lines. Eight cervical cancer cell lines (HeLa229, MS751, ME180, SiHa, HeLa, CasKi, C33A and HCC94) were purchased from the American Type Culture Collection (ATCC; Rockville, MD, USA) and maintained in the Department of Experimental Research, Sun Yat-sen University Cancer Center. They were grown in RPMI-1640 medium (Gibco-BRL, Grand Island, NY, USA) supplemented with $10 \%$ fetal bovine serum (FBS; HyClone Laboratories, Logan, UT, USA) and 1\% antibiotics (100 U/ml penicillin and $100 \mu \mathrm{g} / \mathrm{ml}$ streptomycin).

Samples and patient cohorts. Written informed consent was obtained from each of the cervical cancer patients and the study was approved by the research ethics committee of the Cancer Center of Sun Yat-sen University for the use of these clinical materials for study purposes. The Institutional Review Board (IRB) at the Sun Yat-sen University approved all experimental methods. Included in the present study were tumor specimens from 189 consecutive patients undergoing surgical treatment for cervical cancer at the Department of Sun Yat-sen University Cancer Center from 2006 to 2010. The tumor staging and clinicopathological classification of the tumors were accessed in accordance with the International Federation of Gynecologists and Obstetricians (FIGO, 2009) criteria. The classification of the histological types was based on the World Health Organization (WHO) recommendations.

The 189 patients were in Ib1-IIa2 stage cervical cancer and all received a radical hysterectomy and lymphadenectomy. Patients had not undergone anticancer treatment, such as immunotherapy, chemotherapy, hormone therapy or radiotherapy, before specimen collection. Patients who received postoperative chemotherapy and/or radiotherapy were those with high-risk factors, including: pelvic lymph node metastasis (PLNM), high differentiation grade, positive parametrial involvement, positive lymphovascular space involvement, positive surgical margin, deep stromal invasion and large tumor size $(>4 \mathrm{~cm})$. Patients with only deep stromal invasion or positive surgical margins received postoperative radiotherapy, while patients with only lymphovascular space involvement, high differentiation grade, or large tumor size $(>4 \mathrm{~cm})$ received postoperative chemotherapy. No patients received immunotherapy after surgery.

In total, 189 early-stage cervical cancer patients were included (aged 23-68 years, with a median age of 46 years). The media age is used to determine the age gap of patients. Thus, we separated the groups by a 46-year age gap in the present study. Demographic and clinicopathological information were collected from impatient medical records (Table I). In addition, six pairs of cervical cancer tissues and the matched tumor-adjacent morphologically normal tissues, which were collected from early-stage cervical cancer patients who underwent surgery in 2015 , were frozen in liquid nitrogen at $-80^{\circ} \mathrm{C}$ until further use.

Quantitative and real-time RT-PCR analysis. To explore the role of TIMELESS in the development of cervical cancer, we evaluated its mRNA expression pattern in cervical cancer cell lines and tissues. RNA extraction from the cultured cell lines and surgically dissected tissue samples was carried out using the TRIzol reagent (Invitrogen) following the manufacturer's guidelines. Agilent Bioanalyzer 2100 was used to evaluate the RNA quality (RIN, 2.4-8.8; median 5.9). For PCR-mediated synthesis and amplification of TIMELESS cDNA, an initial amplification reaction using TIMELESS-specific primers was performed with a denaturation step at $95^{\circ} \mathrm{C}$ for $10 \mathrm{~min}$, followed by 30 denaturation cycles at $95^{\circ} \mathrm{C}$ for $60 \mathrm{sec}$, primer annealing at $55^{\circ} \mathrm{C}$ for $30 \mathrm{sec}$, and primer extension at $72^{\circ} \mathrm{C}$ for $30 \mathrm{sec}$. A final extension at $72^{\circ} \mathrm{C}$ for $5 \mathrm{~min}$ was carried out before the reaction was stopped and stored at $4^{\circ} \mathrm{C}$ on completion of the cycling steps. TIMELESS-specific primers were designed using the Primer Express v.2.0 software (Applied Biosystems) as follows: forward 5'-CCATACATCAGTGG ACCAACC-3' and reverse 5'-CTCCTCCGGGCTTCTGA-3'. To control the variability in expression levels, expression data of TIMELESS were normalized to the expression of the housekeeping gene, GADPH. Primers for GADPH were: forward 5'-TTGAGGTCAATGAAGGGGTC-3' and reverse 5'-GAAGGTGAAGGTCGGAGTCA-3'. Quantitative RT-PCR (qRT-PCR) was performed in a total volume of $10 \mu \mathrm{l}$ using the LightCycler 480 instrument (Roche Diagnostics, Penzberg, Germany) with the following conditions: $95^{\circ} \mathrm{C}$ for $5 \mathrm{~min}$, followed by 45 cycles of $95^{\circ} \mathrm{C}$ for $30 \mathrm{sec}, 55^{\circ} \mathrm{C}$ for $30 \mathrm{sec}$ and $72^{\circ} \mathrm{C}$ for $15 \mathrm{sec}$, and a final extension step of $72^{\circ} \mathrm{C}$ for $5 \mathrm{~min}$. The relative quantitative value was expressed by the $2^{-\Delta \Delta \mathrm{Ct}}$ method, where $\mathrm{Ct}$ represents the threshold cycle for each transcript. All experiments were repeated three times.

Western blot analysis. We also evaluated TIMELESS protein expression in cervical cancer cell lines and tissues. The 
Table I. Clinicopathological characteristics and tumor expression of TIMELESS in patients with early-stage cervical cancer.

\begin{tabular}{ll}
\hline Characteristics & No. of cases, (\%) \\
\hline
\end{tabular}

Age (years)
$\leq 46$
$>46$

98 (51.9)

FIGO stage

Ib1

Ib2

IIa1

IIa2

$91(48.1)$

83 (43.9)

28 (14.8)

55 (29.1)

23 (12.2)

Histological type

Squamous carcinoma

$179(94.7)$

Adenocarcinoma

$10(5.3)$

Tumor size, $\mathrm{cm}$

$<4$

$142(75.1)$

47 (24.9)

Squamous cell carcinoma antigen, $\mathrm{ng} / \mathrm{ml}$

$$
\leq 1.5
$$

$>1.5$

$91(48.1)$

98 (51.9)

HPV infection

No

37 (19.6)

Yes

Pelvic lymph node metastasis

No

$134(70.9)$

Yes

55 (29.1)

Tumor recurrence

No

$160(84.7)$

29 (15.3)

Yes

$152(80.4)$

37 (19.6)

Alive

Dead

$111(58.7)$

$16(8.5)$

69 (36.5)

$120(63.5)$

$\geq 1 / 2$

177 (93.7)

$12(6.3)$

$179(94.7)$

Yes

Yes
Table I. Continued.

\begin{tabular}{lc}
\hline Characteristics & No. of cases, $(\%)$ \\
\hline Radiation therapy & $168(88.9)$ \\
No & $21(11.1)$ \\
Yes & \\
Concurrent chemotherapy and radiotherapy & $173(91.5)$ \\
No & $16(8.5)$ \\
Yes & \\
TP chemotherapy & $27(14.3)$ \\
No & $93(49.2)$ \\
Yes & \\
Radical hysterectomy & $9(4.8)$ \\
No & $180(95.2)$ \\
Yes & \\
Expression of TIMELESS protein & $65(34.4)$ \\
Low or none & $124(65.6)$ \\
High &
\end{tabular}

cervical tissues and cell lines were prepared and lysed using the cell total protein extraction kits, on ice, according to the manufacturer's instructions (Millipore, Billerica, MA, USA). Western blots were performed according to standard methods in our previous publication (32). Briefly, we separated equal amounts of protein samples $(30 \mu \mathrm{g})$ on $9 \%$ SDS polyacrylamide gels and transferred them to PVDF membranes (Immobilon P; Millipore, Bedford, MA, USA). Anti-TIMELESS rabbit monoclonal (1:1,000; Abcam, Cambridge, MA, USA) and anti-rabbit (1:2,000; Santa Cruz Biotechnology, Santa Cruz, CA, USA) antibodies were used to detect TIMELESS protein. After detection, the blotted membranes were stripped, and antiGADPH was detected using a mouse monoclonal antibody (1:2,000; Sigma-Aldrich, St. Louis, MO, USA). The secondary antibody (anti-mouse antibody; Santa Cruz Biotechnology) was diluted 1:2,000 in both samples. TIMELESS signals were examined on X-ray film using an ECL prime Western blotting detection reagent (Amersham Biosciences).

Immunohistochemical staining and assessment. Paraffinembedded specimens were obtained from 189 early-stage cervical cancer patients for immunohistochemical analysis. The procedures of immunostaining were carried out with standard protocols. In detail, paraffin tissue slides $(4 \mu \mathrm{m})$ were dried at $60^{\circ} \mathrm{C}$ for $1 \mathrm{~h}$, deparaffinized for $10 \mathrm{~min}$ in xylene (twice), and rehydrated through a series of incubations in graded ethanol solutions $(100,100,95,90$ and $80 \%)$ for $5 \mathrm{~min}$, respectively. Endogenous peroxidase activity was blocked with $3 \%$ hydrogen peroxide in methanol for $15 \mathrm{~min}$ at room temperature to quench endogenous peroxidase activity. Following this, all deparaffinized sections were immersed and boiled in buffered ethylenediaminetetraacetic acid ( $\mathrm{pH}$ 8.0) for $2 \mathrm{~min}$ in a pressure cooker to retrieve antigen, and then cooled to room temperature. Then, sections were immersed with $1 \%$ bovine serum albumin (BSA) to avoid non-specific binding. Subsequently, the slides were immunostained with a primary 

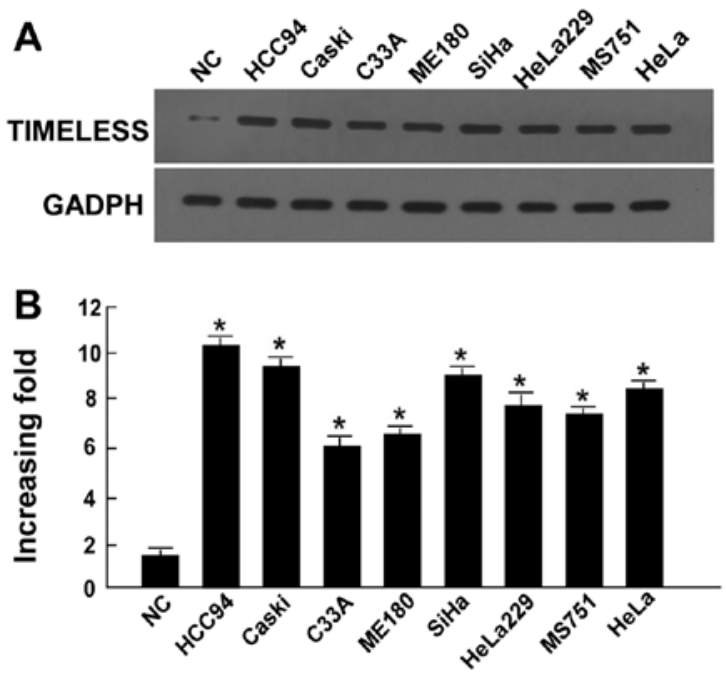

Figure 1. Upregulation of TIMELESS mRNA and protein in cervical cancer cell lines. (A and B) Expression of TIMELESS mRNA and protein in cervical cancer cell lines (HeLa229, MS751, ME180, SiHa, HeLa, CasKi, $\mathrm{C} 33 \mathrm{~A}$ and $\mathrm{HCC} 94)$ and normal cervical tissue were examined by (A) western blotting and (B) qPCR. Expression levels were normalized against GAPDH, respectively. Error bars represent the standard deviation of the mean (SD) calculated from three parallel experiments. ${ }^{*} \mathrm{P}<0.05$.

antibody against TIMELESS (Abcam) diluted at 1:250 at $4^{\circ} \mathrm{C}$ overnight in a moist chamber. Phosphate-buffered saline (PBS) was used as a negative control. After washing with PBS buffer, the slides were incubated with prediluted antirabbit secondary antibody (Abcam) and then incubated with streptavidin-horseradish-peroxidase complex (Abcam). The tissue sections were immersed in 3-amino-9-ethylcarbazole, counterstained with $10 \%$ Mayer's haematoxylin, dehydrated and mounted in Crystal Mount. For visualization, tissue slides were stained with DAB (3,3-diaminobenzidine) for $1 \mathrm{~min}$ at room temperature. Finally, the tissue specimens were counterstained with hematoxylin, dehydrated and mounted using neutral balsam (Sigma-Aldrich).

All immunostained tissue slides were assessed independently by two observers without prior knowledge of clinical data. To evaluate IHC expression of TIMELESS, we applied a scoring system based on multiplying the proportion of positively stained tumor cells and the intensity of staining. The staining intensity (SI) was graded according to the following criteria: 0 , no staining; 1 , weak staining; 2 , modest staining; and 3 , strong staining. The proportion of positive tumor cells was scored as follows: 0 (no positive tumor cells); $1(<10 \%$ positive tumor cells); 2 (10-50\% positive tumor cells); 3 (51-80\% positive tumor cells), and 4 ( $>80 \%$ positive tumor cells). The slides were rescored if the difference between the two pathologists was $>3$. The optimal cut-off values were distinguished and categorized by the median of the immunoreactive score (IRS) results: an SI score of 6 was used to identify tumors with high TIMELESS expression and an SI score of 4 was used for low TIMELESS expression.

Statistical analysis. All calculations were carried out using the SPSS version 18.0 software package (SPSS, Inc., Chicago, IL, USA). The relationship of TIMELESS protein expression levels with clinical and histomorphological characteristics
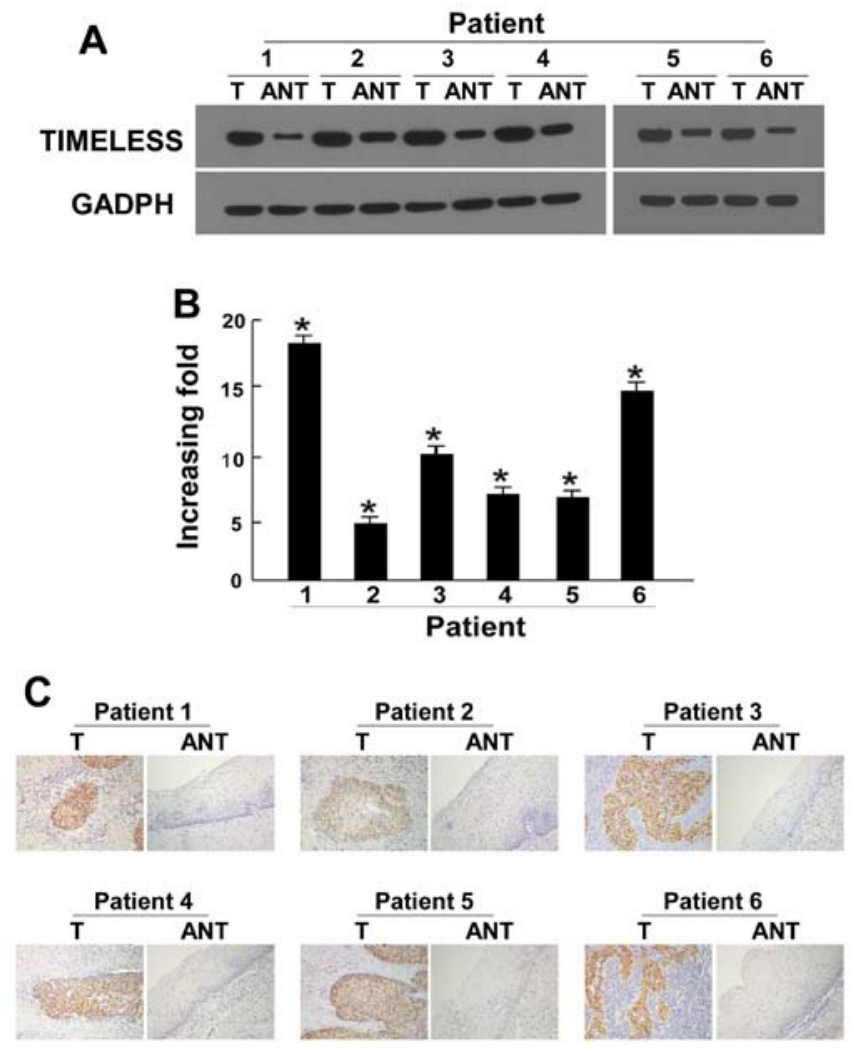

Figure 2. Aberrant expression of TIMELESS mRNA and protein in earlystage cervical cancer tissues. (A) Representative images of western blot analyses of TIMELESS protein expression in six matched pairs of cervical cancer tissue samples (T) and adjacent non-cancerous tissues (ANT). GADPH was used as the loading control. (B) The average T/ANT ratios of TIMELESS mRNA expression in the paired cervical cancer $(\mathrm{T})$ and adjacent non-cancerous tissue sections (ANT) were quantified using qPCR and normalised against GAPDH. The error bars represent the standard deviation of the mean (SD), which was calculated from three parallel experiments. (C) Immunohistochemical staining of TIMELESS protein in six pairs of matched early-stage cervical cancer tissues. ${ }^{*} \mathrm{P}<0.05$.

were determined using a Chi-squared test. The overall survival (OS) and disease-free survival (DFS) curves in association with TIMELESS expression were plotted using the KaplanMeier method and the difference between the groups was tested by a log-rank test. Multivariate analysis was conducted independently for each biomarker, including only significant clinical/histomorphological factors from the univariate analysis. Univariate and multivariate survival analyses were carried out using the Cox regression model. A statistically significant difference was determined by a two-sided P-value of $<0.05$.

\section{Results}

Expression of TIMELESS is upregulated in human cervical cancer. Significantly higher TIMELESS protein expression and mRNA levels were observed in all eight cervical cancer cell lines compared to the normal cervical tissue sample ( $\mathrm{P}<0.05$; Fig. 1). TIMELESS protein expression was also significantly upregulated in the six cervical cancer cases compared with matched adjacent non-cancerous cervical tissues (P<0.05; Fig. 2A). Similarly, qRT-PCR data also indicated that TIMELESS mRNA was significantly upregulated in 

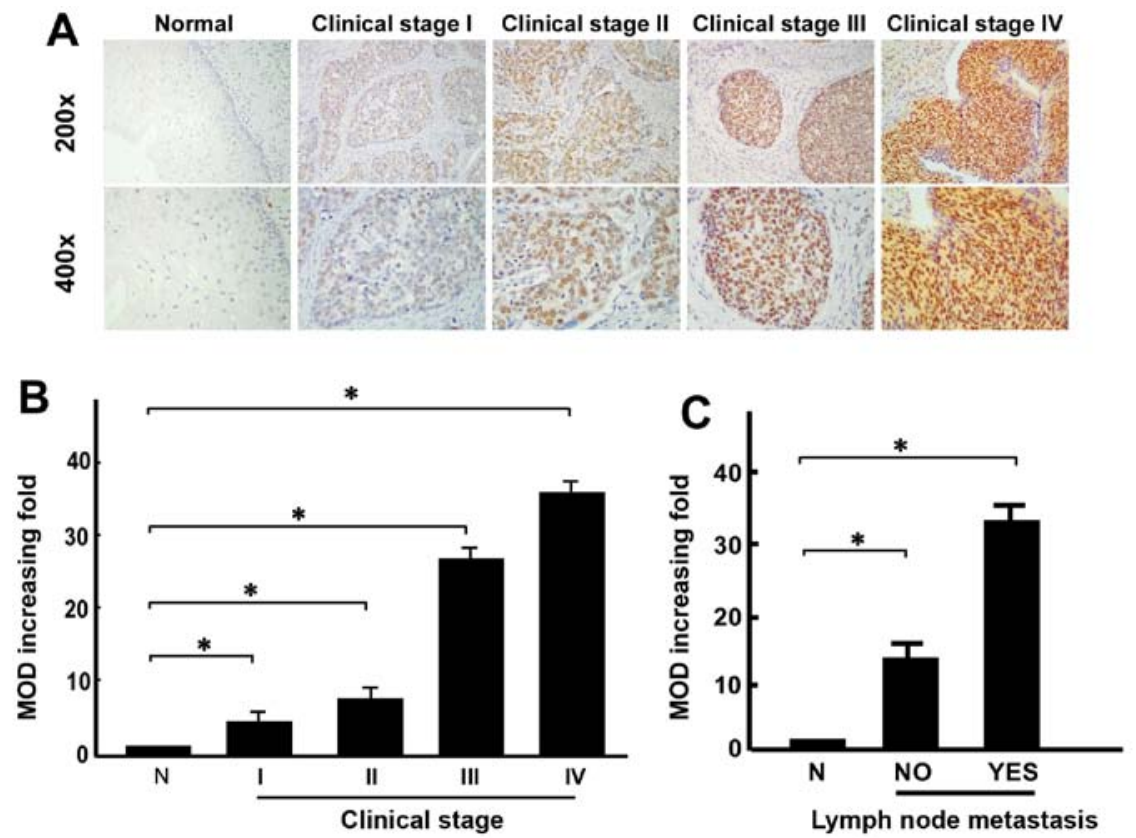

Figure 3. The expression of TIMELESS in cervical cancer tissues from patients at different clinical stages. (A) Representative images from immunohistochemical analyses of TIMELESS protein expression in normal cervical tissues and cervical cancer tissues at different clinical stages. (B) Statistical analyses of the average MOD of TIMELESS staining in normal cervical tissue and cervical cancer specimens at different clinical stages. Values represent the mean \pm SD from three independent experiments. Results are shown as the fold increase in MOD of TIMELESS staining relative to that of normal cervical tissue. (C) The statistical analyses of the average MOD of TIMELESS staining in the lymph node metastasis group and the lymph node metastasis-free group. "P<0.05.

cervical cancer specimens compared to their matched adjacent non-cancerous regions ( $\mathrm{P}<0.05 ;$ Fig. $2 \mathrm{~B})$. By real-time RT-PCR evaluation, the tumor/adjacent non-cancerous $(\mathrm{T} / \mathrm{N})$ ratio of TIMELESS mRNA expression was $>5$-fold in all cases, with the highest ratio being $\sim 18$-fold (Fig. 2B). In agreement with the results of the western blot analysis and real-time RT-PCR assay, IHC showed high expression of TIMELESS protein in cervical cancer lesions (Fig. 2C). Together, our results showed that TIMELESS expression was elevated at both the transcriptional level and translational levels in the cervical cancer cell lines and tissues.

TIMELESS overexpression is correlated with clinicopathological variables of early-stage cervical cancer. We collected 189 paraffin-embedded early-stage cervical cancer tissue samples for IHC, including 83 cases of stage IB1, 28 cases of stage IB2, 55 cases of stage IIA1 and 23 cases of stage IIA2 tumors. Following IHC staining, TIMELESS expression was assessed as positive in 124/189 (65.6\%) cases and weakly positive or negative in 65 cases $(34.4 \%)$. TIMELESS expression was mainly observed in the nucleus and rarely in the cytoplasm of epithelial cells (Fig. 3A). Notably, TIMELESS expression was found in most cancerous lesions in the primary cervical tumors, but was reduced in the adjacent normal cervical regions. TIMELESS protein expression was generally negative in normal cervical tissues, weak in stage IB1 and IB2 cervical cancer tissues, and strong in stage IIA1 and IIA2 cervical cancer tissues; TIMELESS expression obviously increased with the progression of tumor grades $(\mathrm{P}<0.05$; Fig. 3A). Mean optical density (MOD) represents the average response strength of all positive cells in the field of vision. Quantitative IHC data revealed that the MOD values of TIMELESS were significantly associated with the cervical cancer tumor grades
(Fig. 3B), and were higher in cervical cancer tissues with PLNM than in those without PLNM (Fig. 3C).

Next, we examined the relationship between TIMELESS expression and established clinicopathological and investigative parameters using the Chi-squared test and Fisher's exact test (Table II). In patients with early-stage cervical cancer, high TIMELESS expression was markedly associated with age $(\mathrm{P}=0.011)$, clinical stage $(\mathrm{P}<0.001)$, PLNM $(\mathrm{P}<0.001)$, squamous cell carcinoma (SCC) antigen $(\mathrm{P}=0.003)$, tumor recurrence $(\mathrm{P}=0.015)$, vital status $(\mathrm{P}<0.001)$, differentiation grade $(\mathrm{P}<0.001)$, property of surgical margin $(\mathrm{P}=0.036)$ and lymphovascular space involvement $(\mathrm{P}=0.001$; Table III). Moreover, patients with increased TIMELESS protein expression showed strong tendencies to receive postoperative radiotherapy $(\mathrm{P}=0.002)$. However, no significant correlations between TIMELESS expression and the histological type, HPV infection, tumor size, myometrium invasion, or infiltration of the parauterine organ were observed ( $\mathrm{P}>0.05$; Table III). Patients with aberrant TIMELESS protein expression also showed no obvious tendency to receive chemotherapy, concurrent chemotherapy and radiotherapy, chemotherapy with TP regimen (paclitaxel plus cisplatin), or radical hysterectomy ( $\mathrm{P}>0.05$; Table III). TP chemotherapy is the main chemotherapy of cervical cancer patients which combines paclitaxel with cisplatin.

Association coefficient analyses (Spearman's correlation) further confirmed the above-mentioned relationships between the TIMELESS protein expression levels and the clinicopathological features (Table III).

High TIMELESS expression is associated with poor prognosis of patients with early-stage cervical cancer. We examined whether patients with TIMELESS-positive samples had a 
Table II. Correlation between TIMELESS protein expression and the clinicopathological features of early-stage cervical cancer.

\begin{tabular}{|c|c|c|c|c|c|}
\hline Characteristics & Total & $\begin{array}{l}\text { No or weak } \\
\text { TIMELESS } \\
\text { expression }\end{array}$ & $\begin{array}{c}\text { Moderate or strong } \\
\text { TIMELESS } \\
\text { expression }\end{array}$ & $\begin{array}{c}\text { Chi-squared test } \\
\text { (P-value) }\end{array}$ & $\begin{array}{c}\text { Fisher's exact test } \\
\text { (P-value) }\end{array}$ \\
\hline \multicolumn{6}{|l|}{ Age (years) } \\
\hline$\leq 46$ & 98 & $42(22.2)$ & $56(29.6)$ & 0.011 & 0.014 \\
\hline$>46$ & 91 & $23(12.2)$ & $68(36.0)$ & & \\
\hline \multicolumn{6}{|l|}{ Histological type } \\
\hline Adenocarcinoma & 10 & $2(1.1)$ & $8(4.2)$ & 0.325 & 0.498 \\
\hline Squamous cell carcinoma & 179 & $63(33.3)$ & $116(61.4)$ & & \\
\hline \multicolumn{6}{|l|}{ HPV infection } \\
\hline No & 40 & $14(7.4)$ & $23(12.2)$ & 0.623 & 0.700 \\
\hline Yes & 153 & $51(27.0)$ & $101(53.4)$ & & \\
\hline \multicolumn{6}{|l|}{ FIGO stage } \\
\hline Ib1 & 83 & $41(21.7)$ & $42(22.2)$ & $<0.001$ & - \\
\hline Ib2 & 28 & $13(6.9)$ & $15(7.9)$ & & \\
\hline IIa1 & 55 & $7(3.7)$ & $48(25.4)$ & & \\
\hline IIa2 & 23 & $4(2.1)$ & $19(10.1)$ & & \\
\hline \multicolumn{6}{|l|}{ Pelvic lymph node metastasis } \\
\hline Absent & 134 & $60(31.7)$ & $74(39.2)$ & $<0.001$ & $<0.001$ \\
\hline Present & 55 & $5(2.6)$ & $50(26.5)$ & & \\
\hline \multicolumn{6}{|c|}{ Squamous cell carcinoma antigen, $\mathrm{ng} / \mathrm{ml}$} \\
\hline$\leq 1.5$ & 91 & $41(21.7)$ & $50(26.5)$ & 0.003 & 0.004 \\
\hline$>1.5$ & 98 & $24(12.7)$ & $74(39.1)$ & & \\
\hline \multicolumn{6}{|l|}{ Tumor size $(\mathrm{cm})$} \\
\hline$<4$ & 142 & $50(26.5)$ & $92(48.7)$ & 0.680 & 0.726 \\
\hline$\geq 4$ & 47 & $15(7.9)$ & $32(16.9)$ & & \\
\hline \multicolumn{6}{|l|}{ Tumor recurrence } \\
\hline No & 160 & $63(33.3)$ & $106(56.1)$ & 0.015 & 0.014 \\
\hline Yes & 29 & $2(1.1)$ & $18(9.5)$ & & \\
\hline \multicolumn{6}{|l|}{ Vital status (at last follow-up) } \\
\hline Alive & 158 & $63(33.3)$ & $89(47.1)$ & $<0.001$ & $<0.001$ \\
\hline Dead & 35 & $2(1.1)$ & $35(18.5)$ & & \\
\hline \multicolumn{6}{|l|}{ Differentiation grade } \\
\hline G1 & 62 & $38(20.1)$ & $24(12.7)$ & $<0.001$ & - \\
\hline $\mathrm{G} 2$ & 111 & $26(13.8)$ & $85(45.0)$ & & \\
\hline G3 & 16 & $1(0.5)$ & $15(7.9)$ & & \\
\hline \multicolumn{6}{|l|}{ Chemotherapy } \\
\hline No & 84 & $32(16.9)$ & $52(27.5)$ & 0.338 & 0.358 \\
\hline Yes & 105 & $33(17.5)$ & $72(38.1)$ & & \\
\hline \multicolumn{6}{|l|}{ Radiation therapy } \\
\hline No & 168 & $64(33.9)$ & $104(55.0)$ & 0.002 & 0.001 \\
\hline Yes & 21 & $1(0.5)$ & $20(10.6)$ & & \\
\hline \multicolumn{6}{|c|}{ Concurrent chemotherapy and radiotherapy } \\
\hline No & 177 & $62(32.8)$ & $111(58.7)$ & 0.169 & 0.270 \\
\hline Yes & 16 & $3(1.6)$ & $13(6.9)$ & & \\
\hline \multicolumn{6}{|l|}{ TP chemotherapy } \\
\hline No & 27 & $11(9.2)$ & $16(13.3)$ & 0.167 & 0.232 \\
\hline Yes & 93 & $25(20.8)$ & $68(56.7)$ & & \\
\hline
\end{tabular}


Table II. Continued.

\begin{tabular}{|c|c|c|c|c|c|}
\hline Characteristics & Total & $\begin{array}{l}\text { No or weak } \\
\text { TIMELESS } \\
\text { expression }\end{array}$ & $\begin{array}{l}\text { Moderate or strong } \\
\text { TIMELESS } \\
\text { expression }\end{array}$ & $\begin{array}{c}\text { Chi-squared test } \\
\text { (P-value) }\end{array}$ & $\begin{array}{c}\text { Fisher's exact test } \\
\text { (P-value) }\end{array}$ \\
\hline \multicolumn{6}{|c|}{ Radical hysterectomy } \\
\hline No & 9 & $1(0.5)$ & $8(4.2)$ & 0.132 & 0.168 \\
\hline Yes & 180 & $64(33.9)$ & $116(61.4)$ & & \\
\hline \multicolumn{6}{|c|}{ Myometrium invasion } \\
\hline$<1 / 2$ & 69 & $30(15.9)$ & $39(20.6)$ & 0.046 & 0.057 \\
\hline$\geq 1 / 2$ & 120 & $35(18.5)$ & $85(45.0)$ & & \\
\hline \multicolumn{6}{|c|}{ Property of surgical margin } \\
\hline No & 177 & $64(33.9)$ & $113(59.8)$ & 0.036 & 0.037 \\
\hline Yes & 12 & $1(0.5)$ & $11(5.8)$ & & \\
\hline \multicolumn{6}{|c|}{ Infiltration of parauterine organ } \\
\hline No & 179 & $61(32.3)$ & $118(62.4)$ & 0.701 & 0.739 \\
\hline Yes & 10 & $4(2.1)$ & $6(3.2)$ & & \\
\hline \multicolumn{6}{|c|}{ Lymphovascular space involvement } \\
\hline No & 163 & $63(33.3)$ & $100(52.9)$ & 0.001 & 0.001 \\
\hline Yes & 26 & $2(1.1)$ & $24(12.7)$ & & \\
\hline
\end{tabular}

Table III. Spearman correlation analysis of TIMELESS protein expression vs. clinicopathological factors.

\begin{tabular}{lrr}
\hline & \multicolumn{2}{c}{$\begin{array}{c}\text { TIMELESS protein } \\
\text { expression }\end{array}$} \\
\cline { 2 - 3 } & $\begin{array}{c}\text { Spearman's } \\
\text { correlation } \\
\text { coefficient }\end{array}$ & P-value \\
\hline Variables & 0.185 & 0.011 \\
\hline Age & 0.072 & 0.327 \\
Histological type & 0.036 & 0.625 \\
HPV infection & 0.326 & $<0.001$ \\
Clinical staging & 0.341 & $<0.001$ \\
Pelvic lymph node metastasis & 0.216 & 0.003 \\
Squamous cell carcinoma & & \\
antigen, ng/ml & 0.030 & 0.682 \\
Tumor size & 0.177 & 0.015 \\
Recurrence & 0.301 & $<0.001$ \\
Vital status & 0.404 & $<0.001$ \\
Differentiation grade & -0.526 & $<0.001$ \\
Survival time & 0.070 & 0.340 \\
Chemotherapy & 0.221 & 0.002 \\
Radiation therapy & 0.100 & 0.170 \\
Concurrent chemotherapy & & \\
and radiotherapy & 0.126 & 0.169 \\
TP chemotherapy & -0.110 & 0.133 \\
Radical hysterectomy & 0.145 & 0.046 \\
Myometrium invasion & 0.153 & 0.036 \\
Property of surgical margin & -0.028 & 0.703 \\
Infiltration of parauterine organ & 0.232 & 0.001 \\
Lymphovascular space involvement & & \\
\hline
\end{tabular}

poorer survival rate than those with TIMELESS-negative samples. Survival time was markedly different between patients with TIMELESS-positive and TIMELESS-negative samples based on the log-rank test. Patients with higher TIMELESS expression had shorter OS (log-rank, $\mathrm{P}<0.001$; Fig. 4A) and DFS (log-rank, P=0.001; Fig. 4B) than those with lower TIMELESS expression. When examining the whole study cohort, patients with high TIMELESS expression had a significantly lower cumulative 5-year survival rate than those with low TIMELESS protein expression (73.1 vs. 96.8\%, respectively; $\mathrm{P}<0.05$ ).

We also explored the prognostic value of TIMELESS protein expression in different subgroups of patients with early-stage cervical cancer. Subgroups of patients were stratified according to age, HPV infection, FIGO stage, PLNM, SCC antigen, tumor size, differentiation grade, chemotherapy, radiotherapy, concurrent chemotherapy and radiotherapy, TP chemotherapy, radical hysterectomy, myometrium invasion, properties of the surgical margin, infiltration of parauterine organ and lymphovascular space involvement. TIMELESS protein expression was strongly associated with the OS duration of patients aged $>46$ years (log-rank test; $\mathrm{P}=0.008$ ), aged $\leq 46$ years (log-rank test; $\mathrm{P}<0.001$ ), with HPV infection (log-rank test; $\mathrm{P}<0.001)$, without HPV infection (log-rank test; $\mathrm{P}=0.030$ ), without radiotherapy (log-rank test; $\mathrm{P}<0.001$ ), with SCC antigen $\leq 1.5 \mathrm{ng} / \mathrm{ml}$ (log-rank test; $\mathrm{P}=0.004)$, with clinical stage IB1-IB2 (log-rank test; $\mathrm{P}<0.001)$, with TP chemotherapy (log-rank test; $\mathrm{P}=0.005)$, with histological differentiation grade (grades 1 and 2, log-rank test, $\mathrm{P}<0.001$; grades 2 and 3, log-rank test; $\mathrm{P}=0.011$ ), with tumor size (tumor size $<4 \mathrm{~cm}, \log$-rank test; $\mathrm{P}<0.001$; tumor size $\geq 4 \mathrm{~cm}, \log$ rank test; $\mathrm{P}=0.033$ ), with radical hysterectomy (log-rank test; $\mathrm{P}<0.001)$, without infiltration of parauterine organ (log-rank test; $\mathrm{P}<0.001)$, without PLNM (log-rank test; $\mathrm{P}<0.001)$, with 


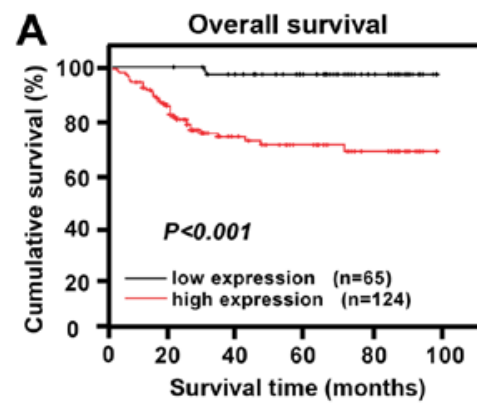

D

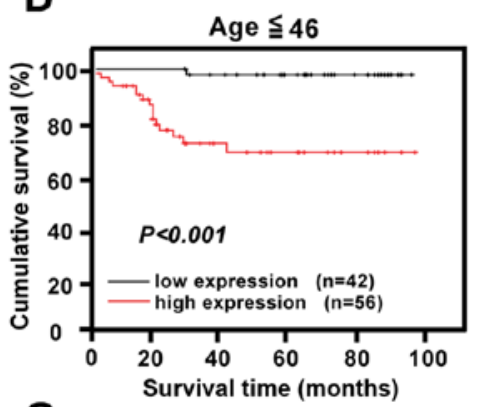

G

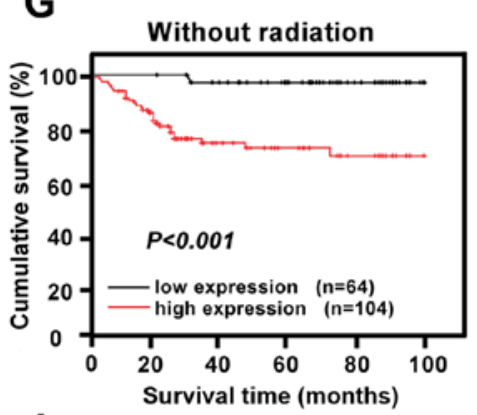

$\mathbf{J}$

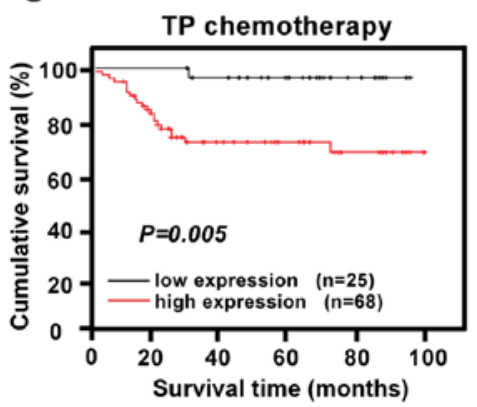

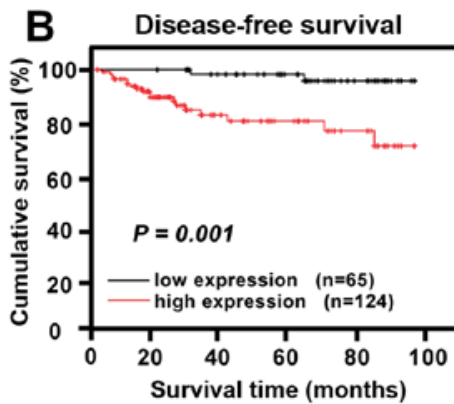

E

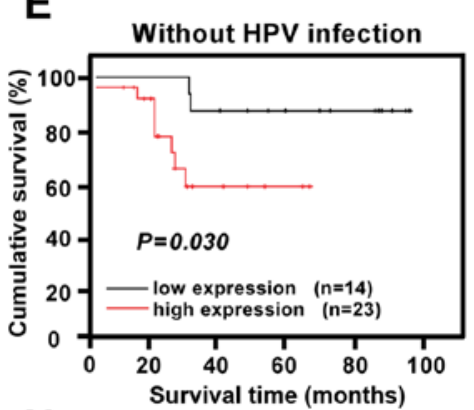

$\mathrm{H}$

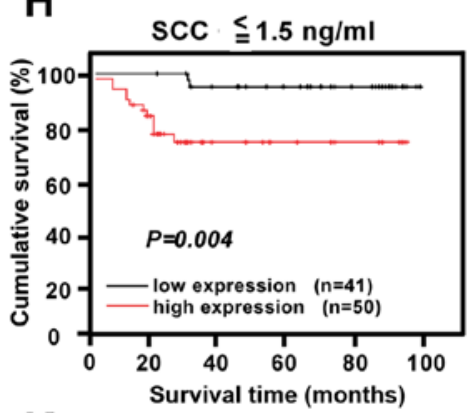

K

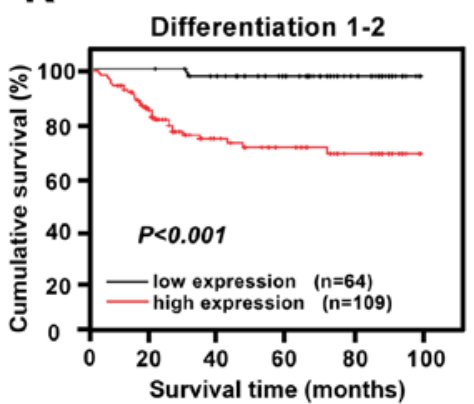

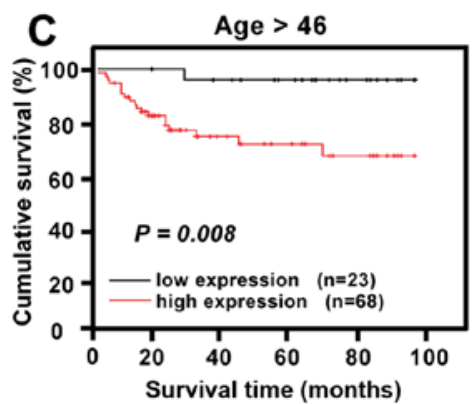

$\mathbf{F}$

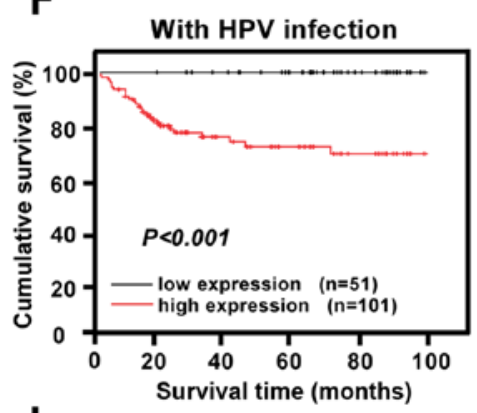

I

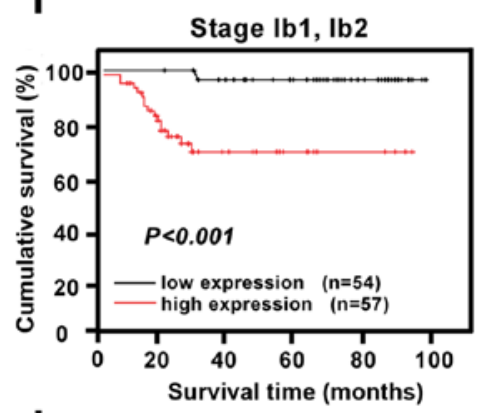

L

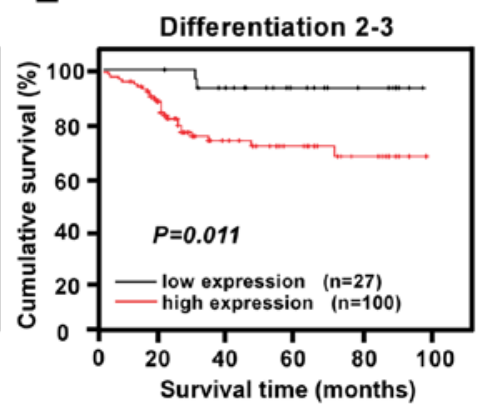

Figure 4. Kaplan-Meier curves of univariate analysis data (log-rank test). The overall survival (OS) and disease-free survival (DFS) for the patients with low TIMELESS expression vs. high TIMELESS expression (A and B). Survival curves for the patients (C) aged $>46$ years, (D) aged $\leq 46$ years, (E) without HPV infection, (F) with HPV infection, (G) without radiation, (H) with SCC $\leq 1.5 \mathrm{ng} / \mathrm{ml}$, (I) at stages Ib1-Ib2, (J) with TP chemotherapy, (K) at differentiation grades 1 and $2,(\mathrm{~L})$ at differentiation grades 2 and 3 . ( $\mathrm{P}<0.05$, respectively).

SCC (log-rank test; $\mathrm{P}<0.001)$, without lymphovascular space involvement (log-rank test; $\mathrm{P}<0.001)$, without properties of the surgical margin (log-rank test; $\mathrm{P}<0.001$ ), without myometrium invasion (log-rank test; $\mathrm{P}=0.001$ ), with myometrium invasion (log-rank test; $\mathrm{P}=0.001)$ and with chemotherapy (log-rank test, $\mathrm{P}<0.001$; Figs. 4 and 5).

Univariate analysis based on the Cox regression model demonstrated that upregulation of TIMELESS protein expression was a significant prognostic biomarker of poor OS $(\mathrm{P}<0.001$; Table IV). In addition, early-stage cervical carcinoma patients with PLNM, with lymphovascular space involvement, and those who had undergone radiation therapy, showed poorer prognosis (Table IV). In our multivariate analysis, only TIMELESS protein expression and tumor recurrence remained significant (Table IV), and served as independent prognostic factors for poor OS in cervical cancer patients $(\mathrm{P}=0.002,95 \% \mathrm{CI}, 2.285-42.639$; and $\mathrm{P}<0.001,95 \%$ CI, 2.430-10.673, respectively).

\section{Discussion}

The present study demonstrated that TIMELESS is abundantly expressed at both transcriptional and translational levels in human cervical cancer compared with normal cervical tissues. This elevation in TIMELESS expression was strongly associated with age, clinical stage, PLNM, 

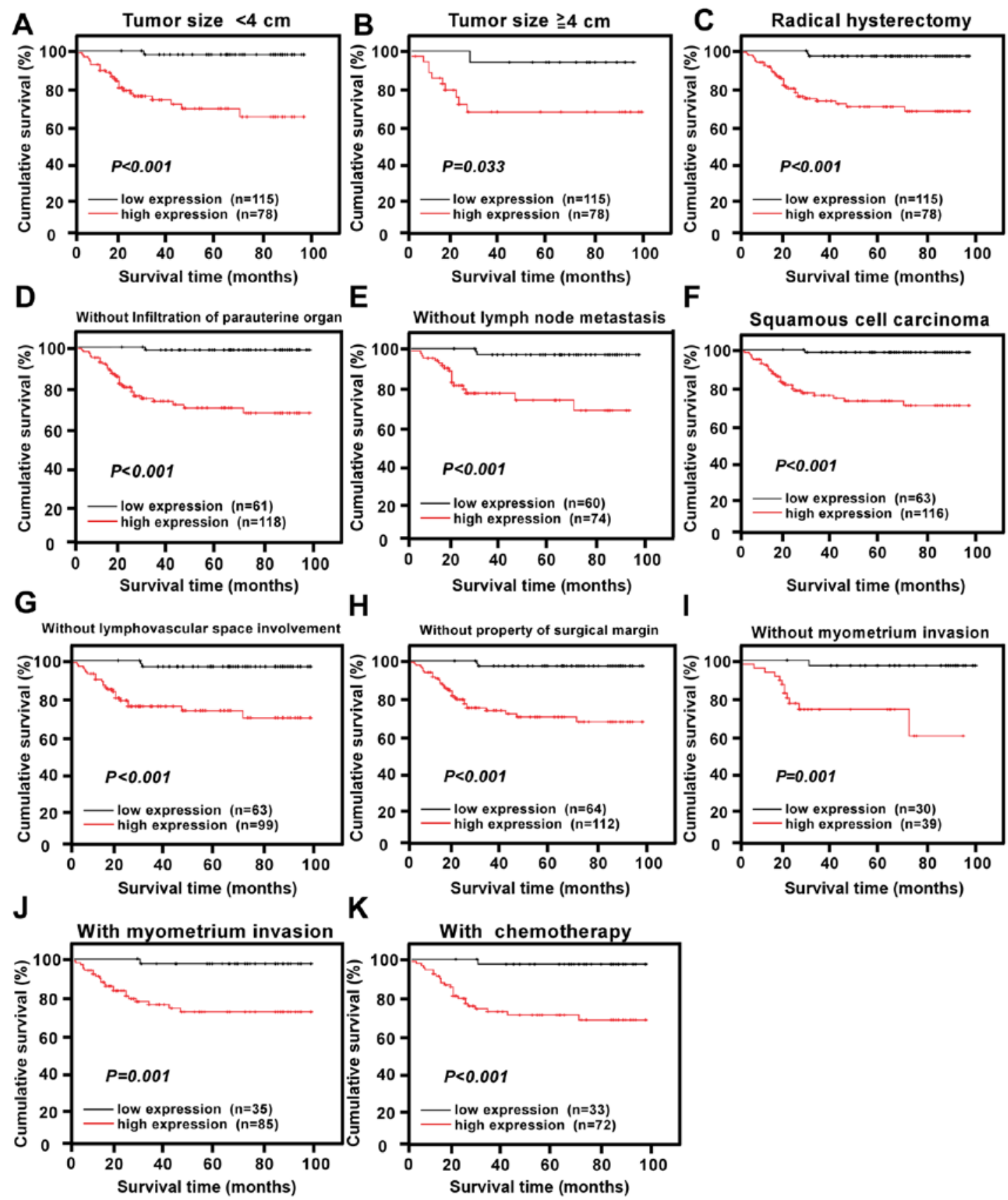

Figure 5. Survival curves for the patients in select patient subgroups (log-rank test). OS rates for patients (A) with tumor size $<4 \mathrm{~cm}$, (B) with tumor size $\geq 4 \mathrm{~cm}$, (C) with radical hysterectomy, (D) without infiltration of parauterine organ, (E) without lymph node metastasis, (F) with pathologic type of squamous cell carcinoma, (G) without lymphovascular space involvement, (H) without property of surgical margin, (I) without myometrium invasion, (J) with myometrium invasion, $(\mathrm{K})$ with chemotherapy. $(\mathrm{P}<0.05$, respectively).

SCC antigen, tumor recurrence, vital status, differentiation grade, property of surgical margin and lymphovascular space involvement. Patients with upregulated TIMELESS protein expression were also more likely to receive postoperative radiotherapy. Furthermore, our survival analysis revealed that high TIMELESS expression was an independent biomarker associated with poorer clinical outcomes in cervical cancer patients.

Similar to our findings, recent reports have shown the importance of TIMELESS in many different types of human cancers (19-31). TIMELESS was overexpressed in a panel of human lung cancer cell lines and clinical lung cancer specimens compared to normal lung controls (19). Associations between TIMELESS and breast cancer risk have also been demonstrated, with TIMELESS being reported as promising marker of tamoxifen resistance in women with estrogen receptor $\alpha$-positive breast tumors (20-22). In colorectal cancer, high TIMELESS expression was significantly associated with the clinical and pathological features (25). TIMELESS expression is also closely related to the clinicopathological factors in bladder cancer (29). On the other hand, TIMELESS is downregulated in kidney cancer cells (28), and TIMELESS transcription was significantly lower in pancreatic ductal adenocarcinoma compared to adjacent tissue and benign tissue (30). As we know, it is quite common that some gene may act as an oncogenic role in several types of cancers while 
Table IV. Univariate and multivariate analyses of prognostic factors in early-stage cervical cancer using a Cox-regression model.

\begin{tabular}{|c|c|c|c|c|c|c|}
\hline & \multicolumn{3}{|c|}{ Univariate analysis } & \multicolumn{3}{|c|}{ Multivariate analysis } \\
\hline & No. of patients & P-value & $\begin{array}{c}\text { Regression } \\
\text { coefficient (SE) }\end{array}$ & P-value & Relative risk & $95 \% \mathrm{CI}$ \\
\hline TIMELESS & & $<0.001$ & $13.712(0.729)$ & 0.002 & 9.871 & $2.285-42.639$ \\
\hline Low expression & 65 & & & & & \\
\hline High expression & 124 & & & & & \\
\hline Pelvic lymph node metastasis & & 0.015 & $2.237(0.330)$ & 0.607 & 1.203 & $0.595-2.434$ \\
\hline Absent & 134 & & & & & \\
\hline Present & 55 & & & & & \\
\hline Radiation therapy & & 0.041 & $2.372(0.422)$ & 0.537 & 1.330 & $0.537-3.291$ \\
\hline No & 168 & & & & & \\
\hline Yes & 21 & & & & & \\
\hline Lymphovascular space involvement & & 0.005 & $2.748(0.360)$ & 0.994 & 0.997 & $0.450-2.209$ \\
\hline No & 163 & & & & & \\
\hline Yes & 26 & & & & & \\
\hline Recurrence & & $<0.001$ & $7.148(0.348)$ & $<0.001$ & 5.092 & $2.430-10.673$ \\
\hline No & 160 & & & & & \\
\hline Yes & 29 & & & & & \\
\hline
\end{tabular}

it plays an anti-oncogenic role in other types of cancers. For example, our previous finding showed that B3GNT3 was upregulated in cervical cancer and is correlated with the progression of cervical cancer (11). Moreover, several reports suggested that B3GNT3 plays an oncogenic role in certain types of cancer such as colon and pancreatic cancer $(33,34)$. However, it is found that B3GNT3 might play a critical role in suppressing the malignant properties of neuroblastoma (35).

With regards to its mechanism of action, TIMELESS appears to regulate the many signaling pathways involved in tumor proliferation, apoptosis, metastasis and the cell cycle. Indeed, TIMELESS has been implicated in cell proliferation in leukemia stem cells (31). It has also been shown to activate the eeF1a2/PI3K/aKt/mtoR axis to contribute to the migratory phenotype in hepatocellular carcinoma, and may also activate the protumorigenic eeF1a2/aKt/mDm4 axis (23). Moreover, TIMELESS was previously shown to be essential for Rad3related-checkpoint kinase 1 (ATR-Chk1)-mediated and ataxia telangiectasia mutated-checkpoint kinase 2 (ATM-Chk2)mediated signaling and S-phase arrest (25). While our results also indicate that TIMELESS is important in the development, progression and metastasis of cervical cancer, further studies are warranted to identify its specific role in the related signaling pathways.

Our results also indicate that overexpression of TIMELESS might be an effective biomarker for identifying patients with more aggressive risk factors, such as those with higher clinical stage, with PLNM, with tumor recurrence, higher differentiation grade, with property of surgical margin and with lymphovascular space involvement. In our experience, several clinical characteristics play important roles in the prognosis of cervical cancer and can affect patients' optimal treatment selections. For example, PLNM has been previously shown to be an important biomarker for the clinical outcome of cervical cancer patients (36), as well as well as for determining the optimal treatment. In the clinic, patients found to have PLNM before surgery will receive chemoradiotherapy instead of undergoing an operation. While many tests (e.g., physical examination, magnetic resonance imaging, biomarkers, among others) are currently available to assess PLNM, they are still prone to errors. Thus, more precise molecular marker are required to avoid misdiagnosis and improper treatment. PLNM also determines the optimal postoperative therapy. For example, after radical hysterectomy plus lymphadenectomy, patients with PLNM will receive postoperative chemotherapy and radiation.

In the present study, we found that TIMELESS expression is clearly associated with PLNM. Thus, TIMELESS may be an effective biomarker for PLNM and for determining the optimal treatment in cervical cancer patients. In previous reports, elevated TIMELESS levels were suggested to play an oncogenic role in hepatocellular carcinoma and promote tumor cell migration (23), and were also associated with tumor metastasis in prostate cancer (24). While our present results are in agreement with these previous studies, we failed to show that TIMELESS is an independent prognostic marker of PLNM, most likely due to the small sample size. In our next study, we aim to detect the utility of TIMELESS as an independent prognostic biomarker of PLNM by examining a larger sample size of early-stage cervical cancer patients. Furthermore, we found a strong correlation between poorer OS and aberrant TIMELESS protein expression in patients without lymph node metastasis. Thus, TIMELESS may be an outstanding marker of PLNM and a good prognostic marker for cervical cancer patients without PLNM, although further studies are required. 
Lymphovascular space involvement has also been identified as an important high-risk factor that affects prognosis and determines postoperative treatment (37). Patients with lymphovascular space involvement may face poorer clinical outcomes and should receive more aggressive treatment, such as postoperative chemotherapy (38). Our data illustrated that TIMELESS protein overexpression is markedly correlated with lymphovascular space involvement and unfavorable survival rates. This indicates that patients with lymphovascular space involvement should undergo more aggressive therapy to reduce the mortality rates.

Past evidence has demonstrated that high differentiation grade is an essential indicator for aggressiveness and worse outcomes in cervical cancer (38). Cervical cancer cells of high differentiation grade show strong proliferative and migratory ability. Furthermore, differentiation grade is critical for the selection of therapy in cervical cancer. According to our treatment guidelines, after resection of the tumor of uterine cervix, patients of high differentiation grade cervical cancer should receive postoperative chemotherapy. Here we showed that the expression of TIMELESS is associated with the differentiation grade of cervical cancer and thus, may be used to evaluate prognosis and guide treatments.

The standard treatments for early-stage cervical cancer patients are currently radical hysterectomy plus lymphadenectomy or chemoradiotherapy. Patients with high-risk factors, such as myometrium invasion, will receive postoperative radiation therapy. On the other hand, patients with high-risk factors, such as lymphovascular space involvement, high grade of tumor differentiation, or a tumor size $>4 \mathrm{~cm}$ will receive postoperative chemotherapy. In addition, patients with PLNM, property of surgical margin, infiltration of the parauterine organ, or lymphovascular space involvement should receive postoperative chemoradiotherapy. Previous reports have proved that postoperative radiation and chemotherapy contribute to the prognosis of patients with high risk factors (39). In the present study, we found that patients with high TIMELESS protein expression showed an increased tendency to receive postoperative radiation therapy. Interestingly, we observed that TIMELESS protein overexpression was significantly correlated with OS in patients with radical hysterectomy, with chemotherapy, with TP chemotherapy, and without postoperative radiation therapy. Thus, TIMELESS protein expression may be useful in identifying patients potentially with shorter OS in these subgroups, who may wish to undertake more aggressive treatments.

While tumor recurrence remains a major issue for cervical cancer mortality (40), it remains difficult to diagnose as the lesions are too small to be seen using available imaging methods. Moreover, no effective techniques or biomarkers are available for predicting tumor recurrence. Our findings demonstrated that TIMELESS protein overexpression is strongly associated with tumor recurrence and is closely correlated with poorer DFS. Thus, TIMELESS protein expression is a useful prognostic biomarker of tumor recurrence in early-stage cervical cancer. Despite our positive results, whether TIMELESS protein or mRNA overexpression could be detected in blood serum and was strongly correlated with tumor recurrence requires further investigation.
Together our results indicate that TIMELESS may be a new therapeutic target for the treatment of cervical cancer. For example, TIMELESS depletion was previously shown to increase sensitivity to doxorubicin, an anticancer drug $(26,27)$. Thus, TIMELESS may represent a novel therapeutic target. However, we must first unravel its exact mechanism of action in cervical cancer to confirm our hypothesis.

In conclusion, our present data demonstrated that TIMELESS expression was upregulated in cervical cancer and was significantly associated with age, clinical stage, PLNM, SCC antigen, tumor recurrence, vital status, differentiation grade, radiation therapy, property of surgical margin and lymphovascular space involvement. Furthermore, TIMELESS protein overexpression was identified as an independent prognostic biomarker for predicting the poor clinical outcome of early-stage cervical cancer patients. However, further investigation into the molecular mechanisms behind the TIMELESS-mediated potential tumorigenic effects in cervical cancer are required.

\section{References}

1. Jemal A, Bray F, Center MM, Ferlay J, Ward E and Forman D: Global cancer statistics. CA Cancer J Clin 61: 69-90, 2011.

2. Ferlay J, Shin HR, Bray F, Forman D, Mathers C and Parkin DM: Estimates of worldwide burden of cancer in 2008: GLOBOCAN 2008. Int J Cancer 127: 2893-2917, 2010.

3. Gu Y, Ma C, Zou J, Zhu Y, Yang R, Xu Y and Zhang Y: Prevalence characteristics of high-risk human papillomaviruses in women living in Shanghai with cervical precancerous lesions and cancer. Oncotarget 7: 24656-24663, 2016.

4. Mayadev J, Lim J, Durbin-Johnson B, Valicenti R and Alvarez E: Smoking decreases survival in locally advanced cervical cancer treated with radiation. Am J Clin Oncol: Jan 22, 2016 (Epub ahead of print).

5. Santesso N, Mustafa RA, Schünemann HJ, Arbyn M, Blumenthal PD, Cain J, Chirenje M, Denny L, De Vuyst H, Eckert LO, et al; Guideline Support Group: World Health Organization Guidelines for treatment of cervical intraepithelial neoplasia 2-3 and screen-and-treat strategies to prevent cervical cancer. Int J Gynaecol Obstet 132: 252-258, 2016.

6. Crafton SM and Salani R: Beyond chemotherapy: An overview and review of targeted therapy in cervical cancer. Clin Ther 38: 449-458, 2016.

7. Sun F, Xiong Y, Zhou XH, Li Q, Xiao L, Long P, Li LJ, Cai MY, Wei YX, Ma YL, et al: Acylglycerol kinase is over-expressed in early-stage cervical squamous cell cancer and predicts poor prognosis. Tumour Biol 37: 6729-6736, 2015.

8. Hou T, Liang D, Xu L, Huang X, Huang Y and Zhang Y: Atypical chemokine receptors predict lymph node metastasis and prognosis in patients with cervical squamous cell cancer. Gynecol Oncol 130: 181-187, 2013.

9. Hou T, Liang D, Yang D, He J, Huang Y and Zhang Y: High expression of CRAM correlates with poor prognosis in patients with cervical carcinoma. Int J Clin Exp Pathol 7: 1060-1068, 2014.

10. Zhang W, Ou J, Lei F, Hou T, Wu S, Niu C, Xu L and Zhang Y: C14ORF166 overexpression is associated with pelvic lymph node metastasis and poor prognosis in uterine cervical cancer. Tumour Biol 37: 369-379, 2015.

11. Zhang W, Hou T, Niu C, Song L and Zhang Y: B3GNT3 Expression is a novel marker correlated with pelvic lymph node metastasis and poor clinical outcome in early-stage cervical cancer. PLoS One 10: e0144360, 2015.

12. Zhang L, Huang H, Zhang L, Hou T, Wu S, Huang Q, Song L and Liu J: URG4 overexpression is correlated with cervical cancer progression and poor prognosis in patients with early-stage cervical cancer. BMC Cancer 14: 885, 2014.

13. Liu L, Xia M, Wang J, Zhang W, Zhang Y and He M: CISD2 expression is a novel marker correlating with pelvic lymph node metastasis and prognosis in patients with early-stage cervical cancer. Med Oncol 31: 183, 2014. 
14. Hou T, Zhang W, Tong C, Kazobinka G, Huang X, Huang Y and Zhang Y: Putative stem cell markers in cervical squamous cell carcinoma are correlated with poor clinical outcome. BMC Cancer 15: 785, 2015

15. Unsal-Kacmaz K, Mullen TE, Kaufmann WK and Sancar A: Coupling of human circadian and cell cycles by the timeless protei. Mol Cell Biol 25: 3109-3116, 2005.

16. Gotter AL, Suppa C and Emanuel BS: Mammalian TIMELESS and Tipin are evolutionarily conserved replication fork-associated factors. J Mol Biol 366: 36-52, 2007.

17. Urtishak KA, Smith KD, Chanoux RA, Greenberg RA, Johnson FB and Brown EJ: Timeless maintains genomic stability and suppresses sister chromatid exchange during unperturbed DNA replication. J Biol Chem 284: 8777-8785, 2009.

18. Stevens RG: Circadian disruption and breast cancer: From melatonin to clock genes. Epidemiology 16: 254-258, 2005.

19. Yoshida K, Sato M, Hase T, Elshazley M, Yamashita R, Usami N Taniguchi T, Yokoi K, Nakamura S, Kondo M, et al: TIMELESS is overexpressed in lung cancer and its expression correlates with poor patient survival. Cancer Sci 104: 171-177, 2013.

20. Fu A, Leaderer D, Zheng T, Hoffman AE, Stevens RG and Zhu Y: Genetic and epigenetic associations of circadian gene TIMELESS and breast cancer risk. Mol Carcinog 51: 923-929, 2012.

21. Dong H, Claffey KP, Brocke S and Epstein PM: Expression of phosphodiesterase 6 (PDE6) in human breast cancer cells. Springerplus 2: 680, 2013.

22. Tozlu-Kara S, Roux V, Andrieu C, Vendrell J, Vacher S, Lazar V, Spyratos F, Tubiana-Hulin M, Cohen P, Dessen P, et al: Oligonucleotide microarray analysis of estrogen receptor a-positive postmenopausal breast carcinomas: Identification of HRPAP20 and TIMELESS as outstanding candidate markers to predict the response to tamoxifen. J Mol Endocrinol 39: 305-318, 2007.

23. Elgohary N, Pellegrino R, Neumann O, Elzawahry HM, Saber MM, Zeeneldin AA, Geffers R, Ehemann V, Schemmer P, Schirmacher P, et al: Protumorigenic role of Timeless in hepatocellular carcinoma. Int J Oncol 46: 597-606, 2015.

24. Chiang YT, Gout PW, Collins CC and Wang Y: Prostate cancer metastasis-driving genes: Hurdles and potential approaches in their identification. Asian J Androl 16: 545-548, 2014.

25. Mazzoccoli G, Panza A, Valvano MR, Palumbo O, Carella M, Pazienza V, Biscaglia G, Tavano F, Di Sebastiano P, Andriulli A, et al: Clock gene expression levels and relationship with clinical and pathological features in colorectal cancer patients. Chronobiol Int 28: 841-851, 2011.

26. Kemp MG, Akan Z, Yilmaz S, Grillo M, Smith-Roe SL, Kang TH, Cordeiro-Stone M, Kaufmann WK, Abraham RT, Sancar A, et al: Tipin-replication protein A interaction mediates Chk1 phosphorylation by ATR in response to genotoxic stress. J Biol Chem 285: 16562-16571, 2010.

27. Yang X, Wood PA and Hrushesky WJ: Mammalian TIMELESS is required for ATM-dependent CHK2 activation and G2/M checkpoint control. J Biol Chem 285: 3030-3034, 2010.

28. Mazzoccoli G, Piepoli A, Carella M, Panza A, Pazienza V, Benegiamo G, Palumbo O and Ranieri E: Altered expression of the clock gene machinery in kidney cancer patients. Biomed Pharmacother 66: 175-179, 2012.
29. Schepeler T, Lamy P, Hvidberg V, Laurberg JR, Fristrup N, Reinert T, Bartkova J, Tropia L, Bartek J, Halazonetis TD, et al: A high resolution genomic portrait of bladder cancer: Correlation between genomic aberrations and the DNA damage response. Oncogene 32: 3577-3586, 2013

30. Relles D, Sendecki J, Chipitsyna G, Hyslop T, Yeo CJ and Arafat HA: Circadian gene expression and clinicopathologic correlates in pancreatic cancer. J Gastrointest Surg 17: 443-450, 2013.

31. Gerber JM, Gucwa JL, Esopi D, Gurel M, Haffner MC, Vala M, Nelson WG, Jones RJ and Yegnasubramanian S: Genome-wide comparison of the transcriptomes of highly enriched normal and chronic myeloid leukemia stem and progenitor cell populations. Oncotarget 4: 715-728, 2013.

32. Zhang W, Niu C, He W, Hou T, Sun X, Xu L and Zhang Y: Upregulation of centrosomal protein 55 is associated with unfavorable prognosis and tumor invasion in epithelial ovarian carcinoma. Tumour Biol 37: 6239-6254, 2015.

33. Shiraishi N, Natsume A, Togayachi A, Endo T, Akashima T, Yamada Y, Imai N, Nakagawa S, Koizumi S, Sekine S, et al: Identification and characterization of three novel beta $1,3-\mathrm{N}$-acetylglucosaminyltransferases structurally related to the beta 1,3-galactosyltransferase family. J Biol Chem 276: $3498-3507,2001$

34. He M, Wu C, Xu J, Guo H, Yang H, Zhang X, Sun J, Yu D, Zhou L, Peng T, et al: A genome wide association study of genetic loci that influence tumour biomarkers cancer antigen 19-9, carcinoembryonic antigen and $\alpha$ fetoprotein and their associations with cancer risk. Gut 63: 143-151, 2014.

35. Ho WL, Che MI, Chou CH, Chang HH, Jeng YM, Hsu WM, Lin $\mathrm{KH}$ and Huang MC: B3GNT3 expression suppresses cell migration and invasion and predicts favorable outcomes in neuroblastoma. Cancer Sci 104: 1600-1608, 2013.

36. Yang S, Liu Y, Xia B, Deng J, Liu T, Li Q, Yang Y, Wang Y, Ning $\mathrm{X}$, Zhang $\mathrm{Y}$, et al: DLL4 as a predictor of pelvic lymph node metastasis and a novel prognostic biomarker in patients with early-stage cervical cancer. Tumour Biol 37: 5063-5074, 2015.

37. Koh WJ, Greer BE, Abu-Rustum NR, Apte SM, Campos SM, Cho KR, Chu C, Cohn D, Crispens MA, Dorigo O, et al: Cervical Cancer, Version 2.2015. J Natl Compr Canc Netw 13: 395-404, quiz 404, 2015.

38. Pol FJ, Zusterzeel PL, van Ham MA, Kuijpers DA, Bulten J and Massuger LF: Satellite lymphovascular space invasion: An independent risk factor in early stage cervical cancer. Gynecol Oncol 138: 579-584, 2015.

39. Lan ML, Yu X, Xiao H, Zhou P, Hu N, Li J and Wang G: Clinical outcomes and toxicity of postoperative intensity-modulated versus three-dimensional conformal radiation therapy in patients with cervical cancer. Asia Pac J Clin Oncol: Feb 28, 2016 (Epub ahead of print).

40. Ikeda Y, Furusawa A, Kitagawa R, Tokinaga A, Ito F, Ukita M, Nomura H, Yamagami W, Tanabe H, Mikami M, et al: Practice patterns of adjuvant therapy for intermediate/high recurrence risk cervical cancer patients in Japan. J Gynecol Oncol 27: e29, 2016. 